\title{
Form-Free Reconstruction of an Electron Energy Distribution Function from Optical Emission Spectroscopy
}

\author{
D. Dodt*, A. Dinklage*, R. Fischer ${ }^{\dagger}$, K. Bartschat ${ }^{* *}$ and O. Zatsarinny** \\ *Max-Planck-Institut für Plasmaphysik, EURATOM Association \\ Wendelsteinstraße 1, 17491 Greifswald, Germany \\ ${ }^{\dagger}$ Boltzmannstraße 2, 85748 Garching, Germany \\ ${ }^{* *}$ Department of Physics and Astronomy, Drake University, Des Moines, IA 50311, USA
}

\begin{abstract}
Spectroscopic data are analyzed by fitting a collisional-radiative model to the emission spectrum of a low-temperature plasma in the wavelength range of visible light. The inference procedure employs Bayesian probability theory and accounts for all measurement and model uncertainties. An effort is made to assign well-justified uncertainties to the atomic data needed for the description of the plasma. The credibility region of the reconstructed electron energy distribution function is obtained by the analysis.
\end{abstract}

Keywords: Integrated Data Analysis, Optical Emission Spectroscopy, Neon, Edge Plasma, Atomic Data

PACS: $52.25 .0 s, 52.20 .-\mathrm{j}$

\section{INTRODUCTION}

Low-temperature plasmas are widely used, e.g., in industrial processes or for lighting purposes [1]. The physics of these discharges is such that the electron gas is the key component of the plasma. The energy dissipation in inelastic collisions and heating processes prevent the electron gas to reach a state of thermal equilibrium. The electron energy distribution function (EEDF) may thus substantially deviate from a Maxwell distribution and has to be described by more complex parameterizations such as the Druyvestein distribution.

Experimentally, EEDFs are usually determined by electrical probe measurements. This approach has the disadvantage of perturbing the plasma being investigated by the electrically biased probe. Moreover, the spatial resolution of probe measurements is limited due to the extent of sheaths appearing close to the probe. Therefore, a noninvasive assessment of EEDFs is attractive both for the validation of probe measurements and physical modeling and for process control.

The approach presented here aims at an assessment of the EEDF from emission spectroscopy. We employ the light emitted from atoms in the gas phase (line emission), which are excited by electron collisions. Since the discharges can be observed with appropriately designed imaging optics, emission spectroscopy may attain high spatial and temporal resolutions [2]. Therefore, the idea to use emission spectroscopy for EEDF assessments is long standing, see e.g. [3]. First attempts to use this approach were based on line-ratio techniques mapping the intensities of different spectral lines onto 
temperatures. For the plasmas under consideration, however, the line intensity ratios are affected by too many processes to infer the EEDF directly. Therefore, the approach used here extends a method described by Fischer and Dose [4]. The extension of the data descriptive model consists in a direct modeling of the raw data, rather than the analysis of pre-analyzed line intensities.

The basic idea is to fit a full physical model to a large number of spectral lines rather than inferring information from a few spectral lines as done in previous approaches. The expected benefit of this method results from the consistent use of correlations in the data. The spectroscopic data to be analyzed are obtained using a cylindrical neon discharge, a well-investigated system with various published results, which can be used for validation $[5,6]$.

\section{DATA DESCRIPTIVE MODEL OF THE SPECTROSCOPIC MEASUREMENT}

The forward model maps the quantity of interest, the EEDF $f_{e}(E)$, onto a simulation of the measured data $\vec{D}$ (spectrometer pixels). It consists of a chain of different elements, which is described below. More details about the data model can be found in [7].

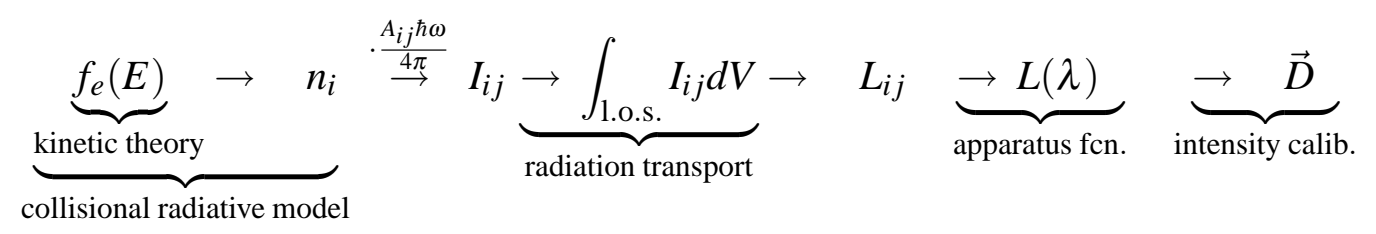

Different parameterizations of the energy distribution are employed to determine electron collision rates for the collisional-radiative model (CRM). EEDFs derived from hybrid modeling of neon discharges, accounting for a kinetic treatment of the electrons $[5,6]$, are used to validate the obtained result.

The CRM consists of a set of balance equations for the population densities $n_{i}$ of 31 excited states of neutral neon taking into account populating and depopulating elementary processes. The locally emitted power $I_{i j}\left[\mathrm{~W} /\left(\mathrm{m}^{3} \cdot \mathrm{sr}\right)\right]$ can be readily obtained by multiplication with the inverse lifetime of the excited states $A_{i j}$ and the photon energy $\hbar \omega$, and division by the full solid angle $(4 \pi)$.

The radiation has to pass through the plasma before it leaves the discharge device. The apparent lifetime of the excited states is affected by the transport of photons if the absorber density is high, i.e., for transitions to the ground state of the atom [8]. Together with the integration along the line of sight (l.o.s.) of the spectrometer, the description of this opacity results in the effective radiance $L_{i j}$ of each transition. The convolution with the apparatus function and a summation over all contributing transitions yields the effective spectral radiance as a function of the wavelength $\lambda$.

The modeling of the actual measurement comprises the translation of $L(\lambda)$ into the detected signals and the mapping of wavelengths to pixel numbers. This requires an absolute intensity calibration, which is obtained using a standard light source. The mapping of pixels to wavelengths is a second-order polynomial, whose parameters are fitted to the data within the reconstruction. 

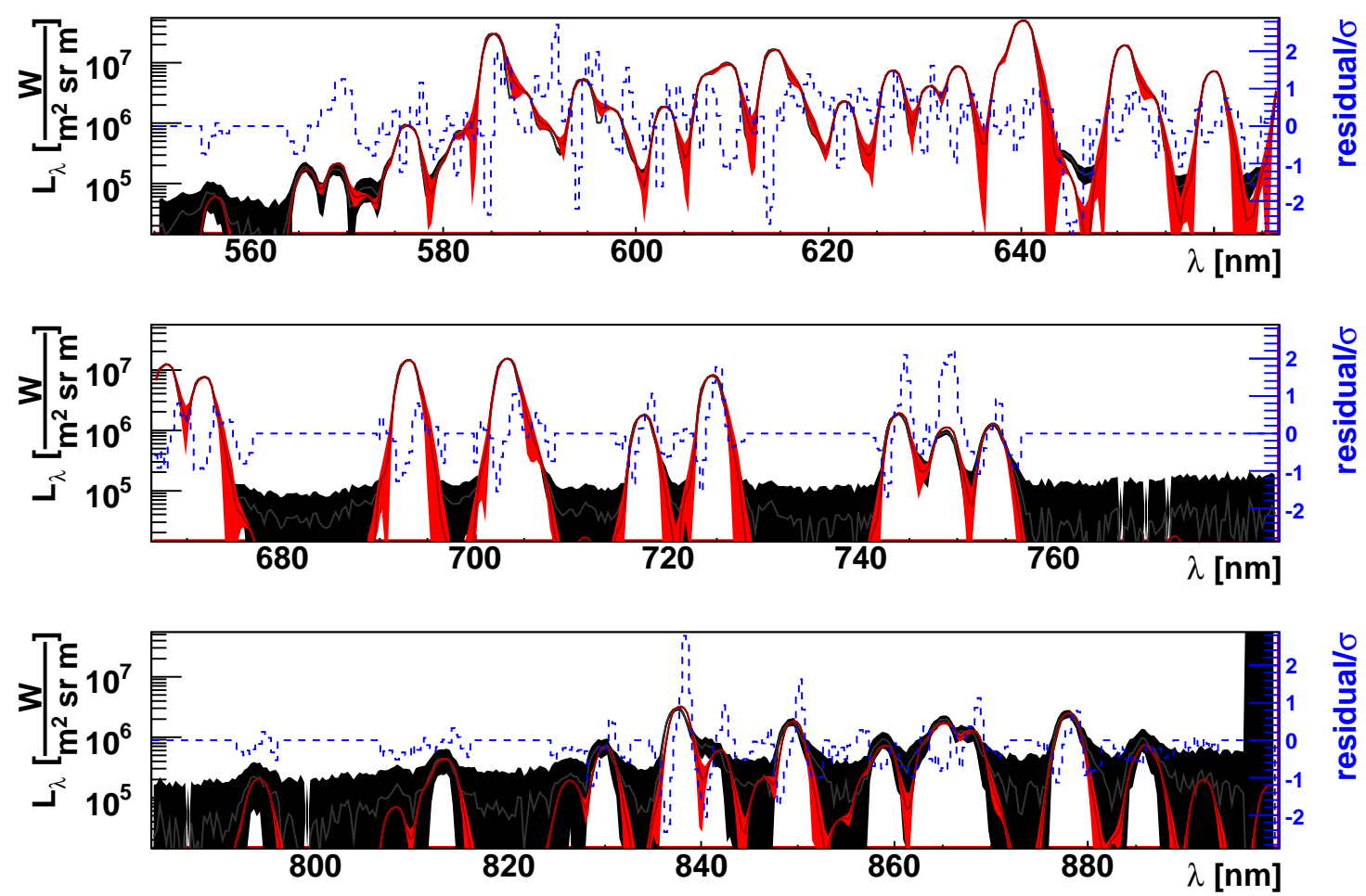

FIGURE 1. Result of the forward model. The intensity is shown as a function of wavelength on a logarithmic scale. The curve inside the light-colored (red) area depicts the modeled spectrum with the uncertainty of the apparatus function. The gray curve in the black area, which is partly hidden behind the modeled spectrum shows the measurement and its uncertainties. The dashed line represents the difference between model and measurement in units of standard deviations.

Figure 1 shows the result of the forward model together with the measurement. Parameters are estimated that yield the best description of the data by minimizing the difference between model $\vec{D}_{\text {sim }}$ and measurement $\vec{D}$ while taking into account additional prior information (see below). The likelihood $P(\vec{D} \mid \vec{\Theta}, I)$, stating the probability to measure a certain spectrum $\vec{D}$ given a parameter set $\vec{\Theta}$, is formulated. Assuming an effective Gaussian error distribution (see also next paragraph) of the $i^{\text {th }}$ pixel of the spectrometer with width $\sigma_{i}$, it is given by

$$
P(\vec{D} \mid \vec{\Theta}, I)=\frac{1}{\prod_{i} \sqrt{2 \pi \sigma_{i}^{2}}} \exp \left\{-\frac{1}{2} \sum_{i} \frac{\left(D_{i}-D_{\text {sim }, i}\right)^{2}}{\sigma_{i}^{2}}\right\} .
$$

Bayes' theorem is used to combine the likelihood of the spectrum with information about model parameters $P(\vec{\Theta})$ not contained in the measurement:

$$
P(\vec{\Theta} \mid \vec{D}, I)=P(\vec{D} \mid \vec{\Theta}, I) \cdot \frac{P(\vec{\Theta})}{P(\vec{D})} .
$$


The resulting probability distribution function (PDF) $P(\vec{\Theta} \mid \vec{D}, I)$ for the parameters of the model is called the posterior distribution, while the PDF $P(\vec{\Theta})$ is called the prior. Together with the marginalization rule quoted below, Bayes' theorem is used to assess parameters entering the model that are not known precisely.

The marginalization theorem for probability distributions allows to integrate out parameters $\vec{\eta}$, called nuisance parameters, which are not parameters of direct interest. However, their uncertainties still affect the uncertainty of the parameters of interest via error propagation:

$$
P(\vec{D} \mid \vec{\Theta}, I)=\int P(\vec{D} \mid \vec{\Theta}, \vec{\eta}, I) d \vec{\eta} .
$$

A table with the relevant nuisance parameters can be found in [9].

\section{Sources of Uncertainty}

The forward model of the measurement may deviate from the outcome of the experiment for various reasons:

Noise: The actual measurement as well as the measurement of the intensity calibration contain electronic noise of the detector chip.

Apparatus Function: The apparatus function is extracted from spectral measurements. An error band describes the reproducibility of the line form at different wavelengths.

Model Parameters: Quantities entering the model may not be known to perfect precision, thereby biasing the modeling result in a systematic way.

Model Assumptions: The plasma model necessarily contains approximations, which may influence the result of the reconstruction.

The noise and the uncertainty of the apparatus function are taken into account by the effective Gaussian error distribution, which is the basis for the likelihood [9, 10]. The effective width $\sigma_{i}$ of the error distribution of the $i^{\text {th }}$ pixel is obtained by adding in quadrature the widths of the noise of the measurement $\sigma_{i, \text { meas }}$ and the uncertainty of the apparatus function $\sigma_{i, \text { app }}$ :

$$
\sigma_{i}=\sqrt{\sigma_{i, \text { meas }}^{2}+\sigma_{i, \mathrm{app}}^{2}}
$$

As mentioned above, the formalism of Bayesian data analysis offers the possibility to treat uncertain model parameters by means of nuisance parameters. This generalization of Gaussian error propagation automatically takes into account correlation effects. The choice of the prior distributions for the atomic data entering the model is discussed in the next paragraph. The other relevant nuisance parameters accounting for experimental uncertainties (wavelength and intensity calibration) or model uncertainties (e.g., some of the escape factors) are listed in [9].

Simplifications of the model may influence the result of the reconstruction. For example, the description of the plasma column with a single CRM for the population densities at the symmetry axis of the discharge and the description of the radiation transport in 
terms of escape factors are important approximations of our model. Therefore a validation of the reconstruction result and its uncertainty margin with independent results are performed to study the applicability of the analysis procedure.

\section{Uncertainties of the Employed Atomic Data}

The collisional-radiative model requires atomic data describing each elementary processes in the plasma. For the electron excitation cross sections and Einstein coefficients, there exists an extensive and consistent dataset from $B$-spline Breit-Pauli $R$-Matrix (BSRM) close-coupling calculations $[11,12]$. These data are combined with those from the atomic line database NIST [13]. The cross sections for ionization of neon in the ground and excited states are taken from [14] and [15].

For the electron-impact excitation cross sections, we make the assumption that the uncertainties can be can be described by a single, energy-independent scale parameter for each cross section. This is a reasonable choice, given that the energy dependence of the cross sections is often known more accurately than the absolute scale (see, for example, ref. [16]), thereby allowing for an efficient implementation in the model. The relative width (root mean-square-variance, rms, divided by expectation value) of the prior distributions for the scale parameters is listed in table 1 . The numbers were chosen according to details of the BSRM calculations and the available independent experimental validations of the cross sections (see $[11,12]$ ).

\begin{tabular}{cc} 
TABLE 1. Uncertainties of the excitation cross sections. \\
\hline final state & $\sigma_{\text {rms }} / \mu$ of log-normal distribution \\
& (relative error) \\
\hline $2 \mathrm{p}^{5} 3 \mathrm{~s} \mathrm{~J}=1$ & $10 \%$ \\
$2 \mathrm{p}^{5} 3 \mathrm{~s} \mathrm{~J}=2$ & $20 \%$ \\
$2 \mathrm{p}^{5} 3 \mathrm{p}$ & $40 \%$ \\
$2 \mathrm{p}^{5} 4 \mathrm{~s}$ & $60 \%$ \\
$2 \mathrm{p}^{5} 3 \mathrm{~d}$ & $60 \%$ \\
\hline
\end{tabular}

The uncertainty of the Einstein coefficients $A_{i j}$ is assessed by considering the results of the BSRM calculations in the length and the velocity form of the electric dipole operator. Since both should, in principle, yield the same result, the difference gives an estimate for the uncertainty. Figure 2 shows the relative difference between the results in the two forms of the dipole operator as a function of the absolute value of the Einstein coefficient. For small $A_{i j}$, the calculations are expected to be less accurate than for stronger transitions. Consequently, the relative width of the prior distribution $\sigma_{\mathrm{rms}} / \mu$ in fig. 2 was chosen depending on the value of $A_{i j}$. It is taken as large as the biggest relative difference for a certain value of $A_{i j}$, but never smaller than $10 \%$. Where both numbers are available, a weighted average of the Einstein coefficients from the BSRM calculations and the NIST database [13] was computed.

For the scale parameters and the Einstein coefficients described above, a log-normal distribution is used as prior. This is a convenient choice when the parameter under consideration may vary over several magnitudes. It is restricted to positive values. For small relative errors, the distribution may be approximated by a Gaussian, while it 


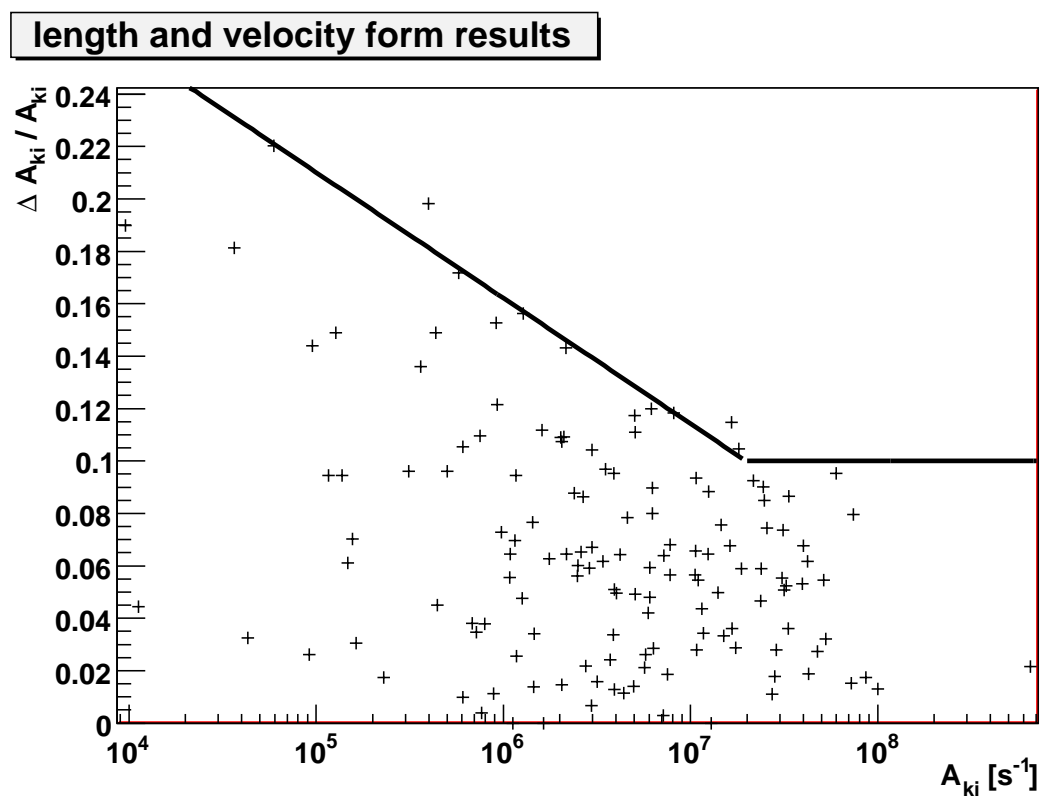

FIGURE 2. The absolute value of the relative difference $2\left|A_{i j,(v)}-A_{i j,(l)}\right| /\left(A_{i j,(v)}+A_{i j,(l)}\right)$ between the BSRM results for the Einstein coefficients in the length and velocity form of the dipole operator is shown as a function of the absolute value of the Einstein coefficient. The straight line depicts the rms-width of the prior distribution as a function of the absolute value of the coefficient (see text).

becomes similar to Jeffrey's prior [17] for larger variances. The PDF of the log-normal distribution is given by

$$
G_{\log }(x \mid \tilde{\mu}, \tilde{\sigma})=\frac{e^{-(\ln x-\tilde{\mu})^{2} /\left(2 \tilde{\sigma}^{2}\right)}}{x \tilde{\sigma} \sqrt{2 \pi}} ; \quad \mu=e^{\tilde{\mu}+\tilde{\sigma}^{2} / 2} ; \quad \sigma_{\mathrm{rms}}=\left(e^{\tilde{\sigma}^{2}}-1\right) e^{2 \tilde{\mu}+\tilde{\sigma}^{2}}
$$

with an expectation value $\tilde{\mu}$ and an rms-variance $\tilde{\sigma}$.

\section{Results and Discussion}

The effect of model assumptions on the EEDF is exemplified in figure 3. Different parameterizations of the EEDF are shown together with the result of hybrid modeling [5]. The latter, which is validated with probe and LIF measurements, acts as a reference. The Maxwellian distribution is shown to demonstrate the deviations from thermal equilibrium. In addition to the Druyvestein parameterization, which describes energy distributions with two temperature parameters, a spline-based parameterization

$$
F_{M}(E)=\underbrace{2 \sqrt{\frac{E}{\pi(k T)^{3}}} \exp \left\{\frac{-E}{k T}\right\}}_{\text {Maxwellian }} \times \exp \left\{f_{\text {Spline }}(E)\right\}
$$



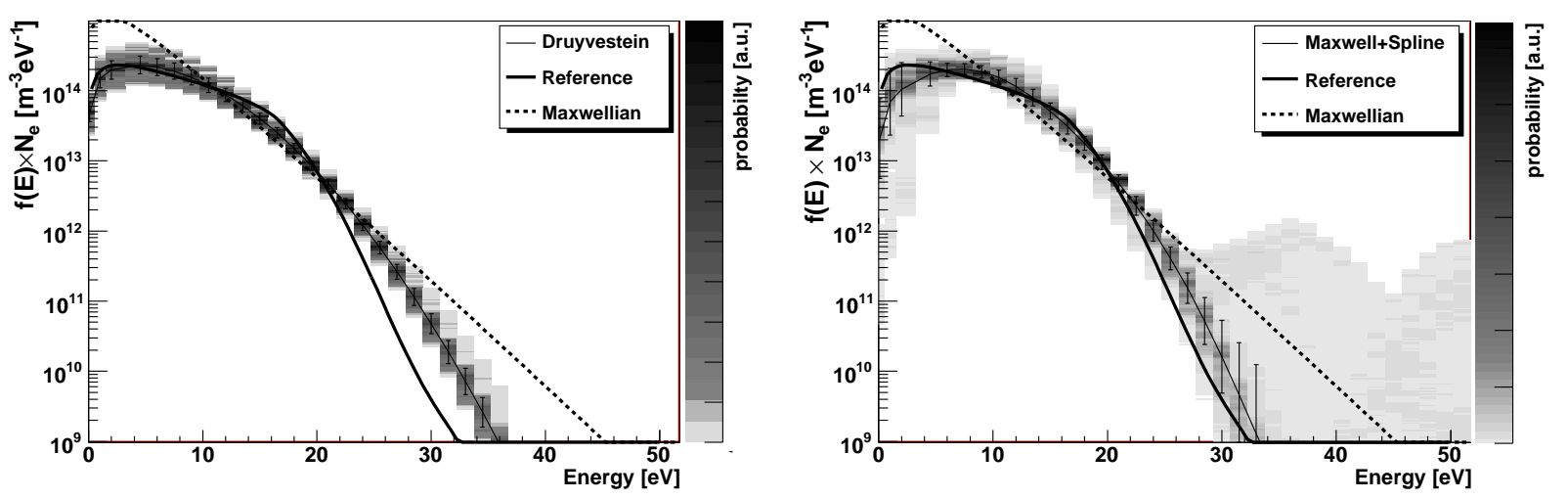

FIGURE 3. The result of the reconstruction is shown for a Druyvestein (left) and for the spline based parameterization described in the text (right). The underlayed histograms show the probability of the EEDF to have a certain value, given the measured data and model assumptions (grey-scale-coded z-axis). The line with error bars shows the expectation value and variance of the logarithm of the EEDF. The Maxwellian and the reference curve (solid curve without error margins), obtained by hybrid modeling of a neon discharge $[5,6]$, is shown for comparison.

is used. The specific choice of the spline $f_{\text {Spline }}(E)$ accounts for the typical scale and form of distribution functions.

The histograms shown in fig 3 depict the posterior distributions, i.e. probability of the EEDF to have a certain value, given the measured data and model assumptions. The histograms are summarized in the overlayed curve with error band, which is obtained by computing the expectation value and variance of the logarithm of the EEDF as a function of energy. The logarithmic scale for the mean value was chosen, because for high energies the posterior has got a flat shape in the logarithm of the EEDF, which corresponds to a strongly asymmetric shape in the linear scale.

For energies below $\approx 25 \mathrm{eV}$, the EEDF reconstruction from the experimental data coincides with the reference distribution. At higher energies, however, the Druyvestein reconstruction deviates from the reference curve while the spline-based reconstruction is more consistent with the reference distribution within the respective error margins. The additional model constraints of the Druyvestein distribution restricts the set of possible EEDFs, thereby leading to a reduced error band. The reference distribution is not compatible with the constraints introduced by the Druyvestein parameterization. In contrast, the spline-based reconstruction does not introduce additional information about the form of the EEDF, therefore it is called form-free parameterization. It can be seen that for energies above $25 \mathrm{eV}$ the posterior distribution flattens out, here the spectroscopic data is not able to determine the value of the EEDF, given the model uncertainties discussed above. The error margins of the spline based parameterization reflect the pure information content of the spectroscopic data. 


\section{ACKNOWLEDGMENTS}

This work was funded by Deutsche Forschungsgemeinschaft through Sonderforschungsbereich Trans Regio 24 (Fundamentals of Complex Plasmas). KB and OZ were supported by the United States National Science Foundation under grants PHY-0244470 and PHY-0555266.

The authors are indebted to V. Dose, R. Preuss, H. Dreier, M. Krychowiak, A. Werner, D. Loffhagen, F. Sigeneger, and D. Uhrlandt for helpful discussions.

\section{REFERENCES}

1. J. Meichsner: Low Temperature Plasmas. In: Plasma Physics: Confinement, Transport and Collective Effects, Springer Lecture Notes Vol. 670, edited by A. Dinklage et al. (Springer, Berlin, 2005)

2. J. Röpcke, P.B. Davies, M. Käning, and B.P. Lavrov: Diagnostics of non-equilibrium molecular plasmas using emission and absorption spectroscopy. In: Low Temperature Plasma Physics, edited by R. Hippler et al. (VCH-Wiley, Berlin, 2001)

3. N. Brenning, J. Phys. D: Appl. Phys. 15, L1 (1982)

4. R. Fischer and V. Dose, Plasma Phys. Control. Fusion 41, 1109 (1999)

5. D. Uhrlandt and S. Franke, J. Phys. D: Appl. Phys. 35, 680 (2002)

6. S. Franke, Diploma Thesis Bilanzgleichungen zur Modelierung der Dynamik einer NeonGlimmentladung, University of Greifswald (Germany, 1996)

7. D. Dodt, A. Dinklage, R. Fischer, and D. Loffhagen, AIP CP 872, 264 (2006)

8. J. E. Lawler and J.J. Curry, J. Phys. D: Appl. Phys. 31, 3235 (1998)

9. D. Dodt, A. Dinklage, and R. Fischer, to be published in AIP CP (2007)

10. V. Dose, R. Fischer, and W. von der Linden, Maximum Entropy and Bayesian Methods, edited by G. Erickson (Kluwer Academic, Dordrecht, 1998).

11. O. Zatsarinny and K. Bartschat, J. Phys. B: At. Mol. Opt. Phys. 37, 2173 (2004)

12. M. Allan, K. Franz, H. Hotop, O. Zatsarinny, and K. Bartschat, J. Phys. B: At. Mol. Opt. Phys. 39, L139 (2006)

13. Yu Ralchenko, F.-C. Jou, D.E. Kelleher, A.E. Kramida, A. Musgrove, J. Reader, W.L. Wiese, and K. Olsen (2007). NIST Atomic Spectra Database (version 3.1.3), [Online]. Available: http://physics.nist.gov/asd3 [2007, October 11]. National Institute of Standards and Technology, Gaithersburg, MD, USA.

14. R. Wetzel, F. Baiocchi, T. Hayes, and R. Freund, Phys. Rev. A 35, 559 (1987)

15. L. Vriens and A. H. M. Smeets, Phys. Rev. A 22, 940 (1980)

16. M. Allan, K. Franz, H. Hotop, O. Zatsarinny, and K. Bartschat, J. Phys. B: At. Mol. Opt. Phys. 39, L139 (2006)

17. D.S. Sivia: Data Analysis: A Bayesian Tutorial, 2nd edition (Oxford University Press, 2006) 